\title{
POLYNOMIALS OF INTERPOLATION AND APPROXIMATION TO MEROMORPHIC FUNCTIONS $\left({ }^{1}\right)$
}

\author{
BY
}

E. B. SAFF

1. Introduction. The object of the present paper is twofold: (1) to establish some general results concerning the convergence and divergence of certain sequences of polynomials which interpolate to a meromorphic function, and (2) to deduce from these results some properties of the polynomials of best uniform approximation to a meromorphic function.

Throughout the paper $E$ shall denote a closed bounded point set in the $z$-plane whose complement $K$ (with respect to the extended plane) is connected and regular in the sense that $K$ possesses a classical Green's function $G(z)$ with pole at infinity. We let $\Gamma_{\rho}(\rho>1)$ denote generically the locus $G(z)=\log \rho$, and denote by $E_{\rho}$ the closed interior of $\Gamma_{\rho}$. Furthermore we set $H(z) \equiv-G_{x}(z)+i G_{y}(z)$.

Suppose the function $f(z)$ is analytic on $E_{\rho}(\rho>1)$ except for a finite number of poles on $\Gamma_{\rho}$ and let $r$ be the highest order of the poles of $f(z)$ on $\Gamma_{\rho}$. Let $P_{n}(z)$ be the polynomial of degree $n$ of best uniform approximation to $f(z)$ on $E$ and set $E_{n}(f)$ $\equiv\left[\max \left|f(z)-P_{n}(z)\right| ; z\right.$ on $\left.E\right]$. In $\S 2$ and $\S 3$ we show that if the point set $E$ has a smooth boundary and $f(z)$ has a pole of order $r$ at some point of $\Gamma_{\rho}$ which is not a critical point of the Green's function $G(z)$, then there exist constants $A_{1}, A_{2}$ such that

$$
0<A_{1} \leqq n^{1-r} \rho^{n} E_{n}(f) \leqq A_{2}<\infty, \quad n>0 .
$$

Inequalities (1) were established by Sewell $[4$, p. 178] for the special case where $E$ is bounded by a single analytic Jordan curve. However, for this configuration $G(z)$ has no critical points and consequently Sewell's methods cannot be applied to the more general point sets $E$ which we shall consider in this paper.

For the case where the function $f(z)$ has poles in critical points we show in $\S 4$ that the inequalities (1) are valid if the exponent of $n$ is replaced by an integer $\lambda$ which depends on the orders of the poles of $f(z)$ and on the multiplicities of the critical points of $G(z)$.

Presented to the Society, April 29, 1968 under the title Interpolation to meromorphic functions; received by the editors January 27, 1969.

$\left.{ }^{1}\right)$ The research of the author was supported (in part) by a Fulbright Grant at the Imperial College of Science and Technology, London, England. The results in $\$ 2$ are part of the author's doctoral dissertation which was directed by Professor J. L. Walsh at the University of Maryland. 
Furthermore, we extend to the geometric setting described above some results of P. Dienes $[2, \S 114]$ on the divergence of the Taylor expansion of a meromorphic function. In particular, we deduce that the best approximating polynomials $P_{n}(z)$ can converge in at most a finite number of points exterior to $\Gamma_{\rho}$.

2. Interpolating polynomials. It is known [1, p. 74] that with the geometric conditions imposed on the point set $E$ there exists a sequence of polynomials $\omega_{n}(z)$ which have respectively $n+1$ zeros and which satisfy for some $\Delta(>0)$

$$
\lim _{n \rightarrow \infty}\left|\omega_{n}(z)\right|^{1 / n}=\Delta \exp G(z),
$$

uniformly for $z$ on each closed bounded set in $K$. Concerning interpolation in the roots of such $\omega_{n}(z)$ we prove

TheOREM 1. Let $E, K, G(z)$, and $H(z)$ be as described in $\$ 1$, and suppose $\omega_{n}(z)$ is a sequence of polynomials having respectively $n+1$ zeros and satisfying (2). If $s_{n, r}(z)$ is the polynomial of degree $n$ which interpolates to the function $(z-\alpha)^{-r}$ in the $n+1$ roots of $\omega_{n}(z)$, where $r$ is a positive integer and $\alpha(\neq \infty)$ lies in $K$, then

$$
\lim _{n \rightarrow \infty} n^{-r} s_{n, r}(\alpha)=H(\alpha)^{r} / r !
$$

Proof. We note that as a consequence of (2) the $\omega_{n}(z)$ have no finite limit point of zeros in $K$, and hence for $n$ sufficiently large no point of interpolation coincides with $\alpha$.

It is clear from the definition of $s_{n, r}(z)$ that

$$
(z-\alpha)^{-r}-s_{n, r}(z)=\omega_{n}(z) p_{n, r}(z) /(z-\alpha)^{r},
$$

where $p_{n, r}(z)$ is a polynomial of degree $r-1$. The equivalence of (4) with

$$
s_{n, r}(z)=\omega_{n}(z)\left(\theta_{n}(z)-p_{n, r}(z)\right) /(z-\alpha)^{r},
$$

where $\theta_{n}(z) \equiv 1 / \omega_{n}(z)$, implies (since $s_{n, r}(z)$ has no finite pole) that $p_{n, r}(z)$ interpolates to $\theta_{n}(z)$ in the point $\alpha$ considered of multiplicity $r$. This property of interpolation is also possessed by the polynomial

$$
q_{n, r}(z) \equiv \sum_{\nu=0}^{r-1} \theta_{n}^{(v)}(\alpha)(z-\alpha)^{v} / \nu !
$$

and since both $p_{n, r}(z)$ and $q_{n, r}(z)$ are of degree $r-1$ we have $p_{n, r}(z) \equiv q_{n, r}(z)$. From (5) it now follows that

$$
(r !) n^{-r} s_{n, r}(\alpha)=n^{-r} \omega_{n}(\alpha) \theta_{n}^{(r)}(\alpha) .
$$

To evaluate the limit as $n \rightarrow \infty$ of the right-hand member of (6) we first note that equation (2) implies

$$
\lim _{n \rightarrow \infty} n^{-1} \omega_{n}^{\prime}(z) \theta_{n}(z)=-H(z),
$$

uniformly on each compact subset of $K$. 
If we assume that for some positive integer $\nu$

$$
\lim _{n \rightarrow \infty} n^{-v} \omega_{n}(z) \theta_{n}^{(v)}(z)=H(z)^{v},
$$

uniformly on each compact subset of $K$, then the convergence of the derived sequence implies

$$
\lim _{n \rightarrow \infty} n^{-1}\left[n^{-v} \omega_{n}(z) \theta_{n}^{(v+1)}(z)+n^{-v} \omega_{n}^{\prime}(z) \theta_{n}^{(v)}(z)\right]=0 .
$$

Since

we thus obtain

$$
\lim _{n \rightarrow \infty}\left(n^{-1} \omega_{n}^{\prime}(z) \theta_{n}(z)\right)\left(n^{-v} \omega_{n}(z) \theta_{n}^{(v)}(z)\right)=-H(z)^{v+1},
$$

$$
\lim _{n \rightarrow \infty} n^{-v-1} \omega_{n}(z) \theta_{n}^{(v+1)}(z)=H(z)^{v+1},
$$

uniformly on each compact subset of $K$.

From (7) it easily follows that equation (8) holds for $\nu=1$. Hence, by induction, it holds for $\nu$ equal to any positive integer and so (3) is a consequence of (6) and (8).

Concerning the degree of approximation to $(z-\alpha)^{-r}$ on $E$ we have

COROLlaRY 1. If $\left|\omega_{n}(z) / \omega_{n}(\alpha)\right| \leqq \varepsilon_{n}$ for $z$ on $E$, then for each positive integer $r$ there exists a constant $A$ independent of $n$ such that

$$
\left|(z-\alpha)^{-r}-s_{n, r}(z)\right| \leqq A n^{r-1} \varepsilon_{n}, \quad z \text { on } E, \quad n>0 .
$$

Proof. From equation (4) we have

$$
(z-\alpha)^{-r}-s_{n, r}(z)=\left[\omega_{n}(z) / \omega_{n}(\alpha)\right] \sum_{\nu=0}^{r-1} \omega_{n}(\alpha) \theta_{n}^{(\nu)}(\alpha)(z-\alpha)^{\nu-r} / \nu !,
$$

and from (8) there follows

$$
\left|\omega_{n}(\alpha) \theta_{n}^{(v)}(\alpha)\right| \leqq A_{0} n^{r-1}, \quad \nu=0,1, \ldots, r-1 .
$$

Hence (9) is a consequence of (10) and the triangle inequality.

It is interesting to note from (4) that the polynomial $s_{n, r}(z)$, uniquely determined by the condition that it interpolate to $(z-\alpha)^{-r}$ in the $n+1$ roots of $\omega_{n}(z)$, also interpolates to $(z-\alpha)^{-r}$ in the roots (if any) of $p_{n, r}(z)$. Regarding the behavior of these additional points of interpolation we prove

Corollary 2. Suppose $H(\alpha) \neq 0$, i.e., $\alpha$ is not a critical point of the Green's function $G(z)$. Then for $n$ sufficiently large each $p_{n, r}(z)$ has precisely $r-1$ zeros, and as $n$ becomes infinite these zeros approach respectively the point $\alpha$.

Proof. From (8) and the representation

we deduce

$$
\omega_{n}(\alpha) p_{n, r}(z)=\sum_{\nu=0}^{r-1} \omega_{n}(\alpha) \theta_{n}^{(v)}(\alpha)(z-\alpha)^{v} / \nu !,
$$

$$
\lim _{n \rightarrow \infty} n^{1-r} \omega_{n}(\alpha) p_{n, r}(z)=H(\alpha)^{r-1}(z-\alpha)^{r-1} /(r-1) !
$$


uniformly for $z$ on each compact subset of the plane. Since the right-hand member of (11) is not identically zero, Hurwitz's Theorem implies that for $n$ sufficiently large each $p_{n, r}(z)$ has at least, and hence precisely, $r-1$ zeros; and these zeros approach the point $\alpha$ considered of multiplicity $r-1$.

Equation (11) is also useful in establishing the following result concerning the divergence of the $s_{n, r}(z)$ (compare [2, p. 480]):

Corollary 3. If $\alpha$ lies on $\Gamma_{\rho}$, then

$$
\lim _{n \rightarrow \infty} \frac{s_{n, r}(z) \omega_{n}(\alpha)}{n^{r-1} \omega_{n}(z)}=\frac{-H(\alpha)^{r-1}}{(r-1) !(z-\alpha)},
$$

uniformly on each closed set exterior to $\Gamma_{\rho}$.

Here and below we interpret $H(\alpha)^{0}$ as unity.

Proof. Let $\rho<\tau<\infty$, and note that (2) implies $\omega_{n}(\alpha) / \omega_{n}(z) \rightarrow 0$ uniformly for $z$ on $\Gamma_{\tau}$. Hence from (11) and the equation

$$
\frac{s_{n, r}(z) \omega_{n}(\alpha)}{n^{r-1} \omega_{n}(z)}=\frac{\omega_{n}(\alpha)}{\omega_{n}(z) n^{r-1}(z-\alpha)^{r}}-\frac{\omega_{n}(\alpha) p_{n, r}(z)}{n^{r-1}(z-\alpha)^{r}}
$$

we deduce that (12) holds uniformly on $\Gamma_{\tau}$. Since the functions which appear in (12) are analytic exterior to $\Gamma_{\tau}$, even at infinity, the limit is uniform as well for $z$ exterior to $\Gamma_{\tau}$, and so the corollary follows from the arbitrariness of $\tau$.

Theorem 1 easily extends to polynomials which interpolate to a meromorphic function of the form

$$
f(z)=g(z)+\sum_{k=1}^{r} B_{k}(z-\alpha)^{-k},
$$

where $\alpha$ lies on $\Gamma_{\rho}$ and $g(z)$ is analytic on $E_{\rho}$. For suppose that the polynomials $\omega_{n}(z)$ of Theorem 1 have no limit point of zeros exterior to $E$, and let $L_{n}(f ; z)$ and $L_{n}(g ; z)$ be the polynomials of degree $n$ which interpolate respectively to the functions $f(z)$ and $g(z)$ in the roots of $\omega_{n}(z)$. Then clearly

$$
L_{n}(f ; z)=L_{n}(g ; z)+\sum_{k=1}^{r} B_{k} s_{n, k}(z)
$$

and since the sequence $L_{n}(g ; \alpha)$ converges [1, p. 154], we deduce from Theorem 1 that

$$
\lim _{n \rightarrow \infty} n^{-r} L_{n}(f ; \alpha)=B_{r} H(\alpha)^{r} / r !
$$

To study the case where $f(z)$ has a pole in more than one point on $\Gamma_{\rho}$ we make the additional assumption that there exists a constant $M$ such that

$$
\left|\omega_{n}(z) / \omega_{n}(t)\right| \leqq M / \rho^{n}, \quad z \text { on } E, t \text { on } \Gamma_{\rho} .
$$

It is known [3] that with certain smoothness conditions on the boundary of $E$, which are discusse? in $\$ 3, \omega_{n}(z)$ exist satisfying both (2) and (13). In particular 
such $\omega_{n}(z)$ exist if $E$ is a finite line segment or if $E$ is bounded by a finite number of mutually exterior analytic Jordan curves.

THEOREM 2. Suppose that the polynomials $\omega_{n}(z)$ of Theorem 1 satisfy (13) and have no limit point of zeros exterior to $E$. Let the function $f(z)$ be analytic on $E_{\rho}$ $(\rho>1)$ except for a finite number of poles on $\Gamma_{\rho}$, and let $r$ be the highest order of the poles of $f(z)$ on $\Gamma_{\rho}$. Then for the polynomials $L_{n}(f ; z)$ of respective degrees $n$ which interpolate to $f(z)$ in the roots of $\omega_{n}(z)$ we have

$$
\left|f(z)-L_{n}(f ; z)\right| \leqq A n^{r-1} / \rho^{n}, \quad z \text { on } E .
$$

Furthermore, if $\alpha$ is a point of $\Gamma_{\rho}$ at which $f(z)$ has a pole of order $r$, then

$$
\lim _{n \rightarrow \infty} n^{-r} L_{n}(f ; \alpha)=B H(\alpha)^{r} / r !
$$

where $B=\lim _{z \rightarrow \alpha}(z-\alpha)^{r} f(z)$.

Here and below constants $A$ are independent of $n$ and $z$ and may change from one inequality to another.

Proof. Let $\alpha=\alpha_{1}, \alpha_{2}, \ldots, \alpha_{m}$ be the distinct poles of $f(z)$ on $\Gamma_{\rho}$ and write $f(z)=g(z)+\sum_{1}^{m} S_{k}(z)$, where $S_{k}(z)$ is the singular part of the pole $\alpha_{k}$. Let $L_{n}(g ; z)$, $L_{n}\left(S_{1} ; z\right), \ldots, L_{n}\left(S_{m} ; z\right)$ be the polynomials of degree $n$ which interpolate respectively to the functions $g(z), S_{1}(z), \ldots, S_{m}(z)$ in the roots of $\omega_{n}(z)$. Since $g(z)$ is analytic on $E_{\rho}$ we have [1, p. 154]

$$
\left|g(z)-L_{n}(g ; z)\right| \leqq A / \rho^{n}, \quad z \text { on } E,
$$

and from (13) and Corollary 1 there follows

$$
\left|S_{k}(z)-L_{n}\left(S_{k} ; z\right)\right| \leqq A n^{r-1} / \rho^{n}, \quad z \text { on } E, k=1, \ldots, m .
$$

Hence the linearity of the interpolation operator together with the triangle inequality imply (14).

To prove the second part of the theorem we first note that by the remarks preceding the statement of Theorem 2 we have

$$
\lim _{n \rightarrow \infty} n^{-r}\left[L_{n}(g ; z)+L_{n}\left(S_{1} ; \alpha\right)\right]=B H(\alpha)^{r} / r !
$$

It is clear from (16) that

$$
\left|\left(z-\alpha_{k}\right)^{r} S_{k}(z)-\left(z-\alpha_{k}\right)^{r} L_{n}\left(S_{k} ; z\right)\right| \leqq A n^{r-1} / \rho^{n}, \quad z \text { on } E,
$$

and since the function whose absolute value appears in the last inequality is a polynomial of degree $n+r$, the generalized Bernstein lemma [1, p. 77] implies that

$$
\left|\left(\alpha-\alpha_{k}\right)^{r} S_{k}(\alpha)-\left(\alpha-\alpha_{k}\right)^{r} L_{n}\left(S_{k} ; \alpha\right)\right| \leqq A n^{r-1} \text {. }
$$

Thus for $k=2, \ldots, m$ we deduce $n^{-r} L_{n}\left(S_{k} ; \alpha\right) \rightarrow 0$, and so (15) follows.

We remark that the second part of Theorem 2 generalizes a result of $P$. Dienes $[2$, p. 479] concerning the divergence of the Taylor expansion of a meromorphic function. 
An extension of Corollary 3 is given in

THEOREM 3. With the hypotheses of Theorem 2, let $\alpha_{1}, \alpha_{2}, \ldots, \alpha_{v}$ be the poles of $f(z)$ on $\Gamma_{\rho}$ which are of order $r$. Then the sequence $L_{n}(f ; z) \omega_{n}\left(\alpha_{1}\right) / n^{r-1} \omega_{n}(z)$ forms a normal family in the exterior of $\Gamma_{\rho}$ and each limit function of the sequence is of the form

$$
\frac{-B_{1} H\left(\alpha_{1}\right)^{r-1}}{(r-1) !\left(z-\alpha_{1}\right)}-\sum_{k=2}^{\nu} \frac{\lambda_{k} B_{k} H\left(\alpha_{k}\right)^{r-1}}{(r-1) !\left(z-\alpha_{k}\right)}
$$

where $B_{k}=\lim _{z \rightarrow \alpha_{k}}\left(z-\alpha_{k}\right)^{r} f(z)$ and the set of possible constants $\lambda_{k}$ is bounded.

Proof. Let $\alpha_{v+1}, \alpha_{v+2}, \ldots, \alpha_{m}$ be the poles of $f(z)$ on $\Gamma_{\rho}$ which are of order less than $r$ and write $f(z)=g(z)+\sum_{k=1}^{m} S_{k}(z)$, where $S_{k}(z)$ is the singular part of the pole $\alpha_{k}$.

Let $\rho<\tau<\infty$, and note that since $g(z)$ is analytic on $E_{\rho}$ there exists a constant $\mu$, $\rho<\mu<\tau$, such that the interpolating polynomials $L_{n}(g ; z)$ are uniformly bounded on $\Gamma_{\mu}$. Thus from the fact [1, p. 67] that $\Gamma_{\tau}=\left(\Gamma_{\mu}\right)_{\tau / \mu}$ and the Bernstein lemma we have $\left|L_{n}(g ; z)\right| \leqq A \tau^{n} / \mu^{n}$ for $z$ on $\Gamma_{\tau}$. There now follows from (2)

$$
\limsup _{n \rightarrow \infty}\left[\max \left|L_{n}(g ; z) \omega_{n}\left(\alpha_{1}\right) / n^{r-1} \omega_{n}(z)\right| ; z \text { on } \Gamma_{\tau}\right]^{1 / n} \leqq \rho / \mu
$$

and since $\tau(>\rho)$ is arbitrary we have $L_{n}(g ; z) \omega_{n}\left(\alpha_{1}\right) / n^{r-1} \omega_{n}(z) \rightarrow 0$ uniformly for $z$ on each closed set exterior to $\Gamma_{\rho}$.

It is clear from Corollary 3 that

$$
\lim _{n \rightarrow \infty} \frac{L_{n}\left(S_{k} ; z\right) \omega_{n}\left(\alpha_{k}\right)}{n^{r-1} \omega_{n}(z)}=\frac{-B_{k} H\left(\alpha_{k}\right)^{r-1}}{(r-1) !\left(z-\alpha_{k}\right)}, \quad k=1,2, \ldots, \nu
$$

and since (13) implies that $\left|\omega_{n}\left(\alpha_{1}\right) / \omega_{n}(z)\right| \leqq M \rho$ for $z$ on $\Gamma_{\rho}$, we also have

$$
\lim _{n \rightarrow \infty} \frac{L_{n}\left(S_{k} ; z\right) \omega_{n}\left(\alpha_{1}\right)}{n^{r-1} \omega_{n}(z)}=0, \quad k=\nu+1, \ldots, m
$$

the limits being uniform on each closed set exterior to $\Gamma_{\rho}$.

Now given any increasing sequence of positive integers we can extract a subsequence $n_{i}$ such that

$$
\lim _{i \rightarrow \infty} \omega_{n_{i}}\left(\alpha_{1}\right) / \omega_{n_{i}}\left(\alpha_{k}\right)=\lambda_{k}, \quad k=2, \ldots, \nu,
$$

and thus

$$
\lim _{i \rightarrow \infty} \frac{L_{n_{i}}\left(S_{k} ; z\right) \omega_{n_{i}}\left(\alpha_{1}\right)}{n_{i}^{r-1} \omega_{n_{i}}(z)}=\frac{-\lambda_{k} B_{k} H\left(\alpha_{k}\right)^{r-1}}{(r-1) !\left(z-\alpha_{k}\right)}, \quad k=2, \ldots, \nu
$$

uniformly for $z$ on each closed set exterior to $\Gamma_{\rho}$. Hence from the linearity of the process of interpolation it follows that each subsequence of $L_{n}(f ; z) \omega_{n}\left(\alpha_{1}\right) / n^{r-1} \omega_{n}(z)$ possesses a subsequence which converges uniformly on each closed set exterior to $\Gamma_{\rho}$ to a function of the form (17), where the constants $\lambda_{k}$ are bounded by $M \rho$. 
It is easy to see from the proof of Corollary 3 that if $r>1$ and $\left|\omega_{n}(\alpha) / \omega_{n}(z)\right| \leqq M_{0}$ for $z$ on $\Gamma_{\rho}$, then the limit (12) also holds for each $z(\neq \alpha)$ on $\Gamma_{\rho}$. Hence a slight modification of the proof of Theorem 3 shows that if $r>1$, then every subsequence of $L_{n}(f ; z) \omega_{n}\left(\alpha_{1}\right) / n^{r-1} \omega_{n}(z)$ possesses a subsequence that converges at each point $z$ on or exterior to $\Gamma_{\rho}, z$ not a pole of $f(z)$ on $\Gamma_{\rho}$, to a function of the form (17).

COROLlary 4. Suppose, in addition to the hypotheses of Theorem 2, that $\alpha_{1}$ is the only pole of highest order $r$ of $f(z)$ on $\Gamma_{\rho}$. Then

$$
\lim _{n \rightarrow \infty} \frac{L_{n}(f ; z) \omega_{n}\left(\alpha_{1}\right)}{n^{r-1} \omega_{n}(z)}=\frac{-B_{1} H\left(\alpha_{1}\right)^{r-1}}{(r-1) !\left(z-\alpha_{1}\right)},
$$

uniformly for $z$ on each closed set exterior to $\Gamma_{\rho}$.

3. Approximating polynomials. We now apply the results of $\$ 2$ to deduce properties of certain sequences of polynomials that approximate a meromorphic function on the point set $E$. Such applications will be justified if we assume that the boundary of $E$ consists of curves of the type described in the following

Definition. A Jordan curve is said to be of class A if it can be represented parametrically in terms of arc length $s$ by $x=x(s), y=y(s)$, where $x(s)$ and $y(s)$ possess second derivatives with respect to $s$ which satisfy a Lipschitz condition of some positive order in $s$.

THEOREM 4. Let $E$ be the closed interior of the mutually exterior Jordan curves $C_{1}, C_{2}, \ldots, C_{m}$, where each $C_{j}$ is of class A. Suppose $f(z)$ is analytic on $E_{\rho}(\rho>1)$ except for a finite number of poles on $\Gamma_{\rho}$ and let $r$ be the highest order of the poles of $f(z)$ on $\Gamma_{\rho}$. Then there exists a sequence of polynomials $p_{n}(z)$ of respective degrees $n$ which satisfy

$$
\left|f(z)-p_{n}(z)\right| \leqq A n^{r-1} / \rho^{n}, \quad z \text { on } E, \quad n>0 .
$$

Furthermore, if $\alpha \in \Gamma_{\rho}$ is a pole of $f(z)$ of order $r$ and $p_{n}(z)$ is any sequence of polynomials of respective degrees $n$ which satisfy (18), then

$$
\lim _{n \rightarrow \infty} n^{-r} p_{n}(\alpha)=B H(\alpha)^{r} / r !
$$

where $B=\lim _{z \rightarrow \alpha}(z-\alpha)^{r} f(z)$.

Proof. Walsh has shown [3, Theorem 1] that for such a point set $E$ there exist polynomials $\omega_{n}(z)$ which have respectively $n+1$ roots and satisfy

$$
\begin{gathered}
\left|\omega_{n}(z)\right| \leqq M_{1} e^{n g}, \quad z \text { on } E, \\
\left|G(z)+g-n^{-1} \log \right| \omega_{n}(z)|| \leqq M_{2} n^{-1},
\end{gathered}
$$

for $z$ on each compact set exterior to $E$, where $e^{g}$ is the capacity of $E$. The roots of the $\omega_{n}(z)$ are chosen so as to be partitioned among the $C_{j}$ and equally distributed on each $C_{j}$. 
It is easy to see from (20) and (21) that the $\omega_{n}(z)$ satisfy both (2) and (13) and hence, by Theorem 2 , the polynomials $L_{n}(f ; z)$ which interpolate to $f(z)$ in the roots of $\omega_{n}(z)$ satisfy (18).

To prove the second part of the theorem assume that polynomials $p_{n}(z)$ of respective degrees $n$ satisfy (18). The triangle inequality implies

$$
\left|p_{n}(z)-L_{n}(f ; z)\right| \leqq A_{1} n^{r-1} / \rho^{n}, \quad z \text { on } E,
$$

and so from the Bernstein lemma we obtain

$$
\left|p_{n}(\alpha)-L_{n}(f ; \alpha)\right| \leqq A_{1} n^{r-1} .
$$

Thus $n^{-r} p_{n}(\alpha)-n^{-r} L_{n}(f ; \alpha) \rightarrow 0$, and so (19) follows from (15).

As a consequence of Theorem 3 we establish

Theorem 5. Let $E, f(z)$, and $r$ be as in Theorem 4 and let $K_{\rho}$ denote the set of points which lie on or exterior to $\Gamma_{\rho}$. Suppose the polynomials $p_{n}(z)$ of respective degrees $n$ satisfy (18). If $r>1$ and if $f(z)$ has a pole of order $r$ at some point $\alpha_{1}$ on $\Gamma_{o}$ which is not a critical point of the Green's function $G(z)$, then

(i) Except for a finite number of points the inequality

$$
\limsup _{n \rightarrow \infty} \frac{\left|p_{n}(z)\right| \rho^{n}}{n^{r-1} \exp (n G(z))}>0
$$

holds for each $z$ on $K_{\rho}$.

(ii) There exists a neighborhood $\Delta$ of $\alpha_{1}$ such that

$$
\liminf _{n \rightarrow \infty} \frac{\left|p_{n}(z)\right| \rho^{n}}{n^{r-1} \exp (n G(z))}>0, \quad z \text { in } \Delta \cap K_{\rho} .
$$

The $p_{n}(z)$ need not be defined for every $n$.

Conclusion (i) implies that the set of points $z$ in $K_{\rho}$ for which the sequence $p_{n}(z)$ converges is finite, and conclusion (ii) implies that $p_{n}(z) \rightarrow \infty$ for $z$ in $\Delta \cap K_{\rho}$.

Proof. As in the proof of Theorem 4 let $L_{n}(f ; z)$ denote the polynomials of respective degrees $n$ which interpolate to $f(z)$ in the $n+1$ roots of the polynomials $\omega_{n}(z)$ which satisfy (20) and (21). For $\mu>1$ we obtain from (20), (21), and (22)

$$
\begin{aligned}
\left|\omega_{n}\left(\alpha_{1}\right) / \omega_{n}(z)\right| & \leqq M_{0} \rho^{n} / \mu^{n}, & & z \text { on } \Gamma_{\mu}, \\
\left|p_{n}(z)-L_{n}(f ; z)\right| & \leqq A_{1} n^{r-1} \mu^{n} / \rho^{n}, & & z \text { on } \Gamma_{\mu},
\end{aligned}
$$

and so for $z$ on or exterior to $\Gamma_{\mu}$ the sequence $\left(p_{n}(z)-L_{n}(f ; z)\right) \omega_{n}\left(\alpha_{1}\right) / n^{r-1} \omega_{n}(z)$ is uniformly bounded, and therefore forms a normal family in the exterior $K$ of $E$. Thus by Theorem 3 the sequence $p_{n}(z) \omega_{n}\left(\alpha_{1}\right) / n^{r-1} \omega_{n}(z)$ forms a normal family in the exterior of $\Gamma_{\rho}$ and each limit function of the sequence is of the form

$$
h(z)-\frac{B_{1} H\left(\alpha_{1}\right)^{r-1}}{(r-1) !\left(z-\alpha_{1}\right)}-\sum_{k=2}^{v} \frac{\lambda_{k} B_{k} H\left(\alpha_{k}\right)^{r-1}}{(r-1) !\left(z-\alpha_{k}\right)},
$$


where $h(z)$ is analytic in $K$, and $\alpha_{1}, \alpha_{2}, \ldots, \alpha_{v}$ are the poles of $f(z)$ on $\Gamma_{\rho}$ which are of order $r$.

Now consider any such limit function $\Phi(z)$. Since $B_{1} H\left(\alpha_{1}\right) \neq 0, \Phi(z)$ has at most a finite number of zeros in $K_{\rho}$. Furthermore by the remarks following the proof of Theorem 3 there exists a subsequence $p_{j}(z) \omega_{j}\left(\alpha_{1}\right) / j^{r-1} \omega_{j}(z)$ which converges to $\Phi(z)$ in $K_{\rho}-$ ppoles of $f(z)$ on $\left.\Gamma_{\rho}\right\}$. Thus if $z \in K_{\rho}$ is neither a pole of $f(z)$ on $\Gamma_{\rho}$ nor a zero of $\Phi(z)$, then

$$
0<\lim _{j \rightarrow \infty}\left|\frac{p_{j}(z) \omega_{j}\left(\alpha_{1}\right)}{j^{r-1} \omega_{j}(z)}\right| \leqq \limsup _{j \rightarrow \infty} \frac{\left|p_{j}(z)\right| A \rho^{j}}{j^{r-1} \exp (j G(z))}
$$

which proves (i).

It is easy to see from (25) and the proof of Theorem 3 that there exists a neighborhood $\Delta$ of $\alpha_{1}$ and a positive constant $\sigma$ such that all limit functions $\Phi(z)$ satisfy $|\Phi(z)| \geqq \sigma$ for $z$ in $\Delta$. If we assume that $\alpha_{1}$ is the only pole of $f(z)$ on $\Gamma_{\rho}$ which lies in $\Delta$, then for fixed $z\left(\neq \alpha_{1}\right)$ in $\Delta \cap K_{\rho}$ we have for $n$ sufficiently large

$$
\left|p_{n}(z) \omega_{n}\left(\alpha_{1}\right) / n^{r-1} \omega_{n}(z)\right| \geqq \sigma / 2>0,
$$

which implies (24). It is obvious from equation (19) that (24) also holds for $z=\alpha_{1}$, and the proof is complete.

We now show that under certain conditions the degree of approximation indicated in (18) is best possible.

THEOREM 6. Let $E, f(z)$, and $r$ be as in Theorem 4 and let $P_{n}(z)$ be the polynomial of degree $n$ which is of best uniform approximation to $f(z)$ on $E$. If $r>1$ and if $f(z)$ has a pole of order $r$ at some point of $\Gamma_{\rho}$ which is not a critical point of $G(z)$, then

$$
n^{1-r} \rho^{n} E_{n}(f) \geqq A>0, \quad n>0,
$$

where $E_{n}(f) \equiv\left[\max \left|f(z)-P_{n}(z)\right| ; z\right.$ on $\left.E\right]$.

Proof. Assume to the contrary that there exists an increasing sequence of integers $j$ for which $j^{1-r} \rho^{j} E_{j}(f) \rightarrow 0$. Let $q(z)$ be the monic polynomial of smallest degree such that $q(z) f(z)$ is analytic on $E_{\rho}$, and let $\pi_{n}(z)$ be the polynomial of degree $n$ which is of best uniform approximation to $q(z) f(z)$ on $E$. From the inequality

$$
\limsup _{n \rightarrow \infty}\left[\max \left|q(z) f(z)-\pi_{n}(z)\right| ; z \text { on } E\right]^{1 / n}<1 / \rho,
$$

it is easy to see that

$$
\left|q(z) P_{j}(z)-\pi_{j}(z)\right| \leqq \sigma_{j} j^{r-1} / \rho^{j}, \quad z \text { on } E,
$$

where $\sigma_{j} \rightarrow 0$. Hence the lemma of Bernstein implies

$$
\left|q(z) P_{j}(z)-\pi_{j}(z)\right| \leqq A \sigma_{j} j^{r-1} \tau^{j} / \rho^{j}, \quad z \text { on } \Gamma_{\tau},
$$

and so if we choose $\tau>\rho$ so that the $\pi_{n}(z)$ are uniformly bounded on $\Gamma_{\imath}$, there follows

$$
\left|P_{j}(z)\right| \rho^{j} / j^{r-1} \tau^{j} \leqq A_{1} \sigma_{j}+A_{2} \rho^{j} \mid j^{r-1} \tau^{j}(\rightarrow 0), \quad z \text { on } \Gamma_{\tau} .
$$


But by Theorem 4 the sequence $j^{1-r} \rho^{j} E_{j}(f)$ is bounded from above and so the last inequality contradicts conclusion (i) of Theorem 5 .

It can be proved directly that if $r=1$, then inequality (26) holds without any restrictions on the location of the poles of $f(z)$ on $\Gamma_{\rho}$. We shall include this result as part of a generalization of Theorem 6 which is proved in the next section.

4. Poles in critical points. Theorem 6 suggests that the degree of approximation indicated in (18) may be improved if the poles of highest order of $f(z)$ coincide with critical points of the Green's function $G(z)$. That this is indeed the case follows from a closer look at inequality (21).

Lemma 1. Let the set $E$ be as in Theorem 4 and suppose the point $\alpha$ in $K$ is a $\nu$-fold critical point of $G(z)$, i.e., $\alpha$ is a zero of order $\nu$ of $H(z)$. Suppose that $\omega_{n}(z)$ is a sequence of polynomials which satisfy (21) for $z$ on each compact subset of $K$, and let $\theta_{n}(z) \equiv 1 / \omega_{n}(z)$. Then for each fixed nonnegative integer $t$ we have the inequalities

$$
\begin{aligned}
\left|\omega_{n}^{(t)}(\alpha) \theta_{n}(\alpha)\right| & \leqq A n^{\lambda}, \\
\left|\omega_{n}(\alpha) \theta_{n}^{(t)}(\alpha)\right| & \leqq A n^{\lambda},
\end{aligned}
$$

where $\lambda=[t /(\nu+1)]$ is the greatest integer less than or equal to $t /(\nu+1)$.

Proof. In order for (21) to hold we make the tacit assumption that the $\omega_{n}(z)$ have no zeros in $K$, and hence the quantities whose absolute values appear in (27) and (28) do indeed exist.

We now proceed by induction on $t$. For $t=0$ inequalities (27) and (28) are obviously valid, so assume they hold for each integer $t$ strictly less than the positive integer $s$.

In order to prove that (27) holds for $t=s$ we estimate the terms on the right-hand side of the identity

$$
\omega_{n}^{(s)}(\alpha) \theta_{n}(\alpha)=\left(\omega_{n}^{\prime}(\alpha) \theta_{n}(\alpha)\right)^{(s-1)}-\sum_{i=1}^{s-1}\left(\begin{array}{c}
s-1 \\
i
\end{array}\right) \omega_{n}^{(s-i)}(\alpha) \theta_{n}^{(i)}(\alpha) .
$$

It is clear that in some closed disk $N(\alpha ; \delta)$ with center $\alpha$ and radius $\delta(>0)$ the function whose absolute value appears in (21) is the real part of an analytic function $f_{n}(z)$ whose derivative is $-H(z)-n^{-1} \omega_{n}^{\prime}(z) \theta_{n}(z)$. From (21) we obtain

$$
0<A_{2} \leqq\left|\exp \left(n f_{n}(z)\right)\right| \leqq A_{1}, \quad z \text { in } N(\alpha ; \delta),
$$

and hence from Cauchy's inequality there follows

$$
A_{2} n\left|f_{n}^{\prime}(z)\right| \leqq\left|n f_{n}^{\prime}(z) \exp \left(n f_{n}(z)\right)\right| \leqq A_{3}, \quad z \text { in } N(\alpha ; \delta / 2) .
$$

The last inequalities imply

$$
\left|H(z)+n^{-1} \omega_{n}^{\prime}(z) \theta_{n}(z)\right| \leqq A_{4} n^{-1}, \quad z \text { in } N(\alpha ; \delta / 2),
$$

and so for fixed $k \geqq 0$ we have

$$
\left|H^{(k)}(\alpha)+n^{-1}\left(\omega_{n}^{\prime}(\alpha) \theta_{n}(\alpha)\right)^{(k)}\right| \leqq A n^{-1} .
$$


Now since $H^{(k)}(\alpha)=0$ for $k=0,1, \ldots, \nu-1$, there follows

$$
\begin{array}{ll}
\left|\left(\omega_{n}^{\prime}(\alpha) \theta_{n}(\alpha)\right)^{(k)}\right| \leqq M, & 0 \leqq k \leqq \nu-1, \\
\left|\left(\omega_{n}^{\prime}(\alpha) \theta_{n}(\alpha)\right)^{(k)}\right| \leqq M n, & \nu \leqq k,
\end{array}
$$

and hence if $\lambda \equiv[s /(\nu+1)]$ we have $\left|\left(\omega_{n}^{\prime}(\alpha) \theta_{n}(\alpha)\right)^{(s-1)}\right| \leqq M n^{\lambda}$.

To estimate the remaining terms on the right-hand side of (29) we let $1 \leqq i \leqq s-1$ and set $\lambda_{1} \equiv[(s-i) /(\nu+1)], \lambda_{2} \equiv[i /(\nu+1)]$. Then by the induction hypothesis we have

$$
\left|\omega_{n}^{(s-i)}(\alpha) \theta_{n}^{(i)}(\alpha)\right|=\left|\omega_{n}^{(s-i)}(\alpha) \theta_{n}(\alpha)\right|\left|\omega_{n}(\alpha) \theta_{n}^{(i)}(\alpha)\right| \leqq A n^{\lambda_{1}+\lambda_{2}} \leqq A n^{\lambda},
$$

and so (27) follows for $t=s$.

It is obvious from the identity

$$
\omega_{n}(\alpha) \theta_{n}^{(s)}(\alpha)=-\omega_{n}^{(s)}(\alpha) \theta_{n}(\alpha)-\sum_{i=1}^{s-1}\left(\begin{array}{c}
s \\
i
\end{array}\right) \omega_{n}^{(s-i)}(\alpha) \theta_{n}^{(i)}(\alpha)
$$

that (28) also holds for $t=s$ and the lemma is proved.

From inequality (28) and the methods used to prove Corollary 1 and Theorem 2 we obtain

THEOREM 7. Let $E$ be the closed interior of a finite number of mutually exterior Jordan curves of class $\mathrm{A}$, and let the sequence of polynomials $\omega_{n}(z)$ which have respectively $n+1$ roots satisfy (20) and (21). Suppose the function $f(z)$ is analytic on $E_{\rho}$ except for poles in the distinct points $\alpha_{1}, \alpha_{2}, \ldots, \alpha_{m}$ on $\Gamma_{\rho}$. Let $r_{k}$ be the order of the pole of $f(z)$ at $\alpha_{k}$ and suppose $G(z)$ has a $\nu_{k}(\geqq 0)$-fold critical point at $\alpha_{k}$. Then the polynomials $L_{n}(f ; z)$ of respective degrees $n$ which interpolate to $f(z)$ in the roots of $\omega_{n}(z)$ satisfy

$$
\left|f(z)-L_{n}(f ; z)\right| \leqq A n^{\lambda} / \rho^{n}, \quad z \text { on } E,
$$

where $\lambda=\max \left\{\left[\left(r_{k}-1\right) /\left(\nu_{k}+1\right)\right] ; k=1, \ldots, m\right\}$.

To obtain an extension of Theorem 3 we prove

Lemma 2. With the hypotheses of Lemma 1 , let $\mu \equiv \nu+1$ and set $\Gamma(\alpha, \nu, k) \equiv$ $(k \mu-1) ! \mu\left(H^{(v)}(\alpha)\right)^{k} /(k-1) !(\mu !)^{k}$. Then for each positive integer $k$ we have

$$
\begin{aligned}
& \lim _{n \rightarrow \infty} n^{-k} \omega_{n}^{(k \mu)}(\alpha) \theta_{n}(\alpha)=(-1)^{k} \Gamma(\alpha, \nu, k), \\
& \lim _{n \rightarrow \infty} n^{-k} \omega_{n}(\alpha) \theta_{n}^{(k \mu)}(\alpha)=\Gamma(\alpha, \nu, k) .
\end{aligned}
$$

Proof. We proceed by induction on $k$. Lemma 1 implies that $\left|\omega_{n}^{(\mu-i)}(\alpha) \theta_{n}^{(i)}(\alpha)\right| \leqq A$ for $i=1, \ldots, \nu$, and from inequality (30) we have $n^{-1}\left(\omega_{n}^{\prime}(\alpha) \theta_{n}(\alpha)\right)^{(v)} \rightarrow-H^{(\nu)}(\alpha)$. Hence from the identities (29) and (31), with $s$ replaced by $\mu$, it follows that (32) and (33) hold for $k=1$. 
Now suppose the lemma is valid for $k=1,2, \ldots, r-1$, and consider the identity (29) with $s$ replaced by $r \mu$. If $i=j \mu+q$ for some integers $j$ and $q$ which satisfy $0 \leqq j \leqq r-1,1 \leqq q \leqq \nu$, then Lemma 1 implies that $\left|\omega_{n}^{(r \mu-i)}(\alpha) \theta_{n}^{(i)}(\alpha)\right| \leqq A n^{r-1}$, and so

$$
\lim _{n \rightarrow \infty} n^{-r} \omega_{n}^{(r \mu-i)}(\alpha) \theta_{n}^{(i)}(\alpha)=0
$$

If $i=j \mu$, where $1 \leqq j \leqq r-1$, it follows from the induction hypothesis that

$$
\lim _{n \rightarrow \infty} n^{-r} \omega_{n}^{(r \mu-i)}(\alpha) \theta_{n}^{(i)}(\alpha)=(-1)^{r-j} \Gamma(\alpha, \nu, r-j) \Gamma(\alpha, \nu, j)
$$

Therefore since inequality (30) implies that $n^{-r}\left(\omega_{n}^{\prime}(\alpha) \theta_{n}(\alpha)\right)^{(r \mu-1)} \rightarrow 0$, we deduce from (29)

$$
\lim _{n \rightarrow \infty} n^{-r} \omega_{n}^{(r \mu)}(\alpha) \theta_{n}(\alpha)=-\sum_{j=1}^{r-1}(-1)^{r-j}\left(\begin{array}{c}
r \mu-1 \\
j \mu
\end{array}\right) \Gamma(\alpha, \nu, r-j) \Gamma(\alpha, \nu, j) .
$$

From the easily verified identity

$$
\left(\begin{array}{c}
r \mu-1 \\
j \mu
\end{array}\right) \Gamma(\alpha, \nu, r-j) \Gamma(\alpha, \nu, j)=\left(\begin{array}{c}
r-1 \\
j
\end{array}\right) \Gamma(\alpha, \nu, r),
$$

we see that the right-hand side of (34) reduces to $(-1)^{r} \Gamma(\alpha, \nu, r)$, and so (32) holds for $k=r$.

It follows from (31) that

$$
\begin{aligned}
\lim _{n \rightarrow \infty} n^{-r} \omega_{n}(\alpha) \theta_{n}^{(r \mu)}(\alpha)= & (-1)^{r+1} \Gamma(\alpha, \nu, r) \\
& -\sum_{j=1}^{r-1}(-1)^{r-j}\left(\begin{array}{c}
r \mu \\
j \mu
\end{array}\right) \Gamma(\alpha, \nu, r-j) \Gamma(\alpha, \nu, j),
\end{aligned}
$$

and so from the identity

$$
\left(\begin{array}{c}
r \mu \\
j \mu
\end{array}\right) \Gamma(\alpha, \nu, r-j) \Gamma(\alpha, \nu, j)=\left(\begin{array}{c}
r \\
j
\end{array}\right) \Gamma(\alpha, \nu, r)
$$

we deduce that (33) also holds for $k=r$ and the lemma is proved.

THEOREM 8. With the hypotheses of Theorem 7 , let $1,2, \ldots, s$ be the values of $k$ for which $\lambda=\left[\left(r_{k}-1\right) /\left(\nu_{k}+1\right)\right]$, and write $r_{k}-1=\lambda\left(\nu_{k}+1\right)+q_{k}$, where $0 \leqq q_{k} \leqq \nu_{k}$. Then the sequence $g_{n}(z) \equiv L_{n}(f ; z) \omega_{n}\left(\alpha_{1}\right) / n^{\lambda} \omega_{n}(z)$ forms a normal family in the exterior of $\Gamma_{\rho}$, and each limit function of the sequence is of the form

$$
\frac{-B \Gamma\left(\alpha_{1}, \nu_{1}, \lambda\right)}{\left(\lambda \nu_{1}+\lambda\right) !\left(z-\alpha_{1}\right)^{q_{1}+1}}-\sum_{i=1}^{q_{1}} \frac{A_{1 i}}{\left(z-\alpha_{1}\right)^{i}}-\sum_{k=2}^{s} \sum_{i=1}^{q_{k}+1} \frac{A_{k i}}{\left(z-\alpha_{k}\right)^{i}}
$$

where $B=\lim _{z \rightarrow \alpha_{1}}\left(z-\alpha_{1}\right)^{r_{1}} f(z)$ and the set of possible constants $A_{k i}$ is bounded.

Furthermore if $\lambda>0$, then each subsequence of $g_{n}(z)$ possesses a subsequence which converges to a function of the form (35) at each point $z$ on or exterior to $\Gamma_{\rho}, z$ not a pole of $f(z)$ on $\Gamma_{\rho}$. 
The proof of Theorem 8, which follows from Lemma 2 and the methods used in $\S 2$, is left to the reader.

Since the first term of expression (35) is not identically zero, the reasoning used to establish Theorem 5 yields

THeOREM 9. Let $E, f(z)$, and $\lambda$ be as in Theorem 7 and let $D_{\rho}$ denote the exterior of $\Gamma_{\rho}$. If the polynomials $p_{n}(z)$ of respective degrees $n$ satisfy

$$
\left|f(z)-p_{n}(z)\right| \leqq A n^{\lambda} / \rho^{n}, \quad z \text { on } E,
$$

then

(i) Except for a finite number of points the inequality

$$
\limsup _{n \rightarrow \infty} \frac{\left|p_{n}(z)\right| \rho^{n}}{n^{\lambda} \exp (n G(z))}>0
$$

holds for each $z$ in $D_{\rho}$.

(ii) If for the integers $r_{k}$ and $\nu_{k}$, which correspond to the pole $\alpha_{k}$, we have $\lambda=$ $\left[\left(r_{k}-1\right) /\left(v_{k}+1\right)\right]$, then there exists a neighborhood $\Delta$ of $\alpha_{k}$ such that

$$
\liminf _{n \rightarrow \infty} \frac{\left|p_{n}(z)\right| \rho^{n}}{n^{\lambda} \exp (n G(z))}>0, \quad z \text { in } \Delta \cap D_{\rho} .
$$

Furthermore if $\lambda>0$, then conclusion (i) holds with $D_{\rho}$ replaced by $D_{\rho}+\Gamma_{\rho}$, and conclusion (ii) holds with $D_{\rho}$ replaced by $D_{\rho}+\Gamma_{\rho}-\left\{\alpha_{k}\right\}$.

The $p_{n}(z)$ need not be defined for every $n$.

We conclude with a result which states that the degree of approximation indicated in (36) is best possible.

THEOREM 10. Let $E, f(z)$, and $\lambda$ be as in Theorem 7, and let

$$
E_{n}(f) \equiv\left[\max \left|f(z)-P_{n}(z)\right| ; z \text { on } E\right],
$$

where $P_{n}(z)$ is the polynomial of degree $n$ which is of best uniform approximation to $f(z)$ on $E$. Then there exist constants $A_{1}$ and $A_{2}$ such that

$$
0<A_{2} \leqq n^{-\lambda} \rho^{n} E_{n}(f) \leqq A_{1}<\infty, \quad n>0 .
$$

The fact that the sequence $\varepsilon_{n} \equiv n^{-\lambda} \rho^{n} E_{n}(f)$ is bounded from above follows immediately from Theorem 7 . To establish that $\varepsilon_{n} \geqq A_{2}>0$ one shows that the contrary assumption would violate conclusion (i) of Theorem 9.

We remark that the results of $\S 3$ and $\S 4$, which apply to the polynomials of best approximation in the Tchebycheff norm, can be extended so as to apply to polynomials of best approximation in other norms, such as the $q$ th power line integral norm taken over the boundary of $E$. These extensions are reserved for another occasion. 


\section{REFERENCES}

1. J. L. Walsh, Interpolation and approximation, 3rd ed., Amer. Math. Soc. Colloq. Publ., Vol. 20, Amer. Math. Soc., Providence, R. I., 1960.

2. P. Dienes, The Taylor series, Dover, New York, 1957.

3. J. L. Walsh, A sequence of rational functions with application to approximation by bounded analytic functions, Duke Math. J. 30 (1963), 177-190.

4. W. E. Sewell, Degree of approximation by polynomials in the complex domain, Ann. of Math. Studies No. 9, Princeton Univ. Press, Princeton, N. J., 1942.

\section{IMPERIAL COLLEGE, \\ LONDON, ENGLAND}

